DOI: http://dx.doi.org/10.12957/demetra.2015.14141

\title{
Perfil nutricional de crianças menores de cinco anos que frequentaram um berçário institucional
}

\section{Nutritional status of children under five years old who attended an institutional nursery}

\author{
Andressa Gomes Barroa Felizola? \\ Rita de Cássia Coelho de Almeida Akutsu' \\ Amanda Branquinho Silva' \\ Karin Eleonora de Oliveira Sávio \\ 1 Universidade de Brasilia, Faculdade de Ciências \\ da Saúde, Departamento de Nutrição. Brasília- \\ DF, Brasil. \\ Correspondência / Corrrespondence \\ Andressa Gomes Barros Felizola \\ E-mail:andressagbarros@gmail.com
}

\section{Resumo}

Objetivo: Avaliar o perfil nutricional de crianças menores de cinco anos que frequentaram um berçário institucional no Distrito Federal. Métodos: A amostra foi composta por crianças que frequentaram o berçário institucional entre março de 2010 e dezembro de 2013. Foram analisados dados socioeconômicos, ambientais, demográficos, biológicos e antropométricos de mães e crianças, e aplicado um Questionário de Frequência Alimentar para analisar o consumo alimentar infantil. Para avaliação da qualidade global da dieta, foi utilizado um escore baseado no Recommended Foods Score (Contagem de Alimentos Recomendados). Resultados: A amostra foi composta por 26 mães e 28 crianças. Quanto às mães, 67,9\% estavam na faixa etária de 31 a 40 anos, 67,9\% eram pós-graduadas, 85,7\% recebiam mais de 10 salários, $75 \%$ eram casadas, $85,7 \%$ eram eutróficas e $14,3 \%$ com sobrepeso. A média de idade das crianças foi de 29,44 meses $( \pm 14,18) ; 67,9 \%$ permaneceram em aleitamento materno exclusivo até seis meses e $50 \%$ foram desmamadas entre 12 e 15 meses; $88,9 \%$ das crianças estavam eutróficas e $11,1 \%$, com risco de sobrepeso; 28,6\% apresentaram consumo de açúcares e bebidas açucaradas acima da recomendação; $32,2 \%$, consumo de frutas abaixo do recomendado e $67,9 \%$, consumo de hortaliças abaixo do recomendado pela Sociedade Brasileira de Pediatria; 10,7\% realizavam as refeições assistindo televisão quase sempre e 21,4\%, nunca. A nota média da dieta foi $23,25 \pm 2,31$ pontos de um total de 28. Conclusão: A frequência a um berçário institucional pode contribuir de forma positiva para o estado nutricional e os hábitos alimentares da criança.

Palavras-chave: Perfil Nutricional. Crianças. Consumo de Alimentos. Antropometria. Berçários Institucionais. 


\section{Abstract}

Aim: To evaluate the nutritional status of children under five years who attended an institutional nursery in the Federal District, Brazil. Methods: The sample consisted of children who attended the institutional nursery between March 2010 and December 2013. The children and their mothers' socioeconomic, environmental, demographic, anthropometric and biological data were analyze and a Food Frequency Questionnaire was applied in order to evaluate the children's food intake. To evaluate the diet's overall quality, we used a score based on the Recommended Foods Score. Results: The sample consisted of 26 mothers and 28 children. As for the mothers, $67.9 \%$ were aged 31 to 40 years; $67.9 \%$ had postgraduate degrees; $85.7 \%$ received more than 10 minimum wages; $75 \%$ were married; $85.7 \%$ were normal weight and $143 \%$ overweight. As for the children, their mean age was 29.44 months ( \pm 14.18 ); $67.9 \%$ were on exclusive breastfeeding up to 6 months and 50\% were weaned between 12 and 15 months; $88.9 \%$ of children were eutrophic and $11.1 \%$ had an overweight risk; $28.6 \%$ presented consumption of sugars and sugary drinks above the recommended; $32.2 \%$ presented consumption of fruit below the recommended and $67.9 \%$ a vegetable consumption below the recommended by the Brazilian Society of Pediatrics; $10.7 \%$ often had meals watching television and $21.4 \%$ never. The diet's average score was $23.25 \pm 2.31$ points out of a total of 28 . Conclusion: Attending an institutional nursery can contribute positively to the nutritional status and children's eating habits.

Key words: Nutritional Status. Children. Food Consumption. Anthropometry. Institutional Nurseries.

\section{Introdução}

Um número cada vez maior de crianças precisa passar parte do tempo em berçários ou creches. No Brasil, do ano 2000 para 2010 houve aumento de 14\% na frequência de crianças de 0 a 3 anos e 28,7\% de crianças de $4-5$ anos em creches. ${ }^{1}$ Isso decorre possivelmente, dentre outros fatores, da crescente inserção das mulheres no mercado de trabalho.

Nesse contexto, o berçário e a creche ganham especial destaque, pois é nos primeiros anos de vida que os hábitos alimentares são estabelecidos. Eles são influenciados por diversos fatores de caráter genético, ambiental e psicológico que estão sujeitos a 
modificações. Existem predisposições genéticas para preferências por certos alimentos em detrimento de outros, porém as experiências durante as fases da vida são capazes de moldá-las. ${ }^{2}$

Há muitos fatores que contribuem para a definição dos hábitos alimentares como aleitamento materno, introdução alimentar, influência dos pais, contexto social e afetivo e influência da mídia. ${ }^{3} \mathrm{O}$ foco deste trabalho é o aleitamento materno, o consumo alimentar e o estado nutricional.

O aleitamento materno é recomendado por dois anos ou mais para a criança e deve ser exclusivo até os seis meses. O aleitamento materno exclusivo (AME) é suficiente para suprir as necessidades nutricionais e imunológicas, além de contribuir para aumentar o vínculo afetivo entre mãe e bebê, favorecer o ganho de peso adequado e prevenir diversas infecções, doenças e mortes infantis. ${ }^{4}$

Segundo a pesquisa sobre Aleitamento Materno nas Capitais Brasileiras, ${ }^{5}$ a prevalência de aleitamento materno exclusivo em menores de seis meses no Distrito Federal foi de $50 \%$, e do aleitamento materno complementar até um ano, de 65,4\%. A pesquisa também revela uma progressão em relação à prevalência do aleitamento materno exclusivo de 1999 a 2008. No Distrito Federal, em 1999, a mediana era de 45 dias; em 2008, houve elevação para 77,7 dias, ou seja, a evolução foi de $72,67 \%$. $^{6}$

Além disso, o aleitamento materno tem a capacidade de transmitir sabores provenientes dos alimentos ingeridos pela mãe, favorecendo uma melhor aceitabilidade posterior dos alimentos pela criança. Sendo assim, se as escolhas da mãe forem saudáveis, a criança terá mais facilidade de aceitar tais alimentos. ${ }^{7}$

Após os seis meses de idade, as necessidades nutricionais da criança mudam, tornandose necessária a introdução complementar de uma alimentação saudável e adequada, com o intuito de suprir tais demandas. ${ }^{4}$ Esse é um momento importante na vida da criança, pois contribuirá para a formação de seus hábitos alimentares, seu crescimento e desenvolvimento. O profissional que acompanha esse processo tem o papel de enfatizar a relevância dessa fase e dar apoio e orientação necessária para que a adesão das mães seja a maior possível. ${ }^{8}$

No Brasil, o consumo alimentar de crianças de seis a 59 meses é caracterizado pela alta ingestão de alimentos ricos em gorduras, sal e açúcar como refrigerantes, salgadinhos, frituras e doces. Em paralelo, é baixo o consumo de alimentos saudáveis como hortaliças, vegetais e carne. ${ }^{9}$

Esses dados confirmam a ocorrência da transição alimentar e nutricional que vem ocorrendo no mundo. Houve uma mudança tanto no padrão alimentar quanto no 
estado nutricional da população. Se antes o cenário principal era a desnutrição, agora a preocupação é com o sobrepeso e a obesidade. A transição nutricional é caracterizada pelo consumo elevado de alimentos com alta densidade energética, mas pobres em nutrientes, em detrimento da baixa ingestão de alimentos ricos em fibras, vitaminas e minerais como frutas, hortaliças e alimentos integrais, além da diminuição da prática de exercícios físicos. Como consequência, é possível observar o desenvolvimento de doenças relacionadas a carências ou distúrbios nutricionais, como anemia e deficiência de vitaminas e minerais, além do aumento de doenças crônicas não transmissíveis como diabetes, hipertensão e obesidade..$^{10}$

Também contribui para a formação dos hábitos alimentares e, consequentemente, no estado nutricional a influência da mídia sobre as crianças. A televisão, por meio de propaganda e anúncios, influencia a escolha do alimento pela criança, estimulando o consumo de alimentos industrializados que, em regra, têm alto teor calórico, são ricos em açúcar e gordura, além de baixa concentração de vitaminas e minerais. ${ }^{11}$ Por isso, é importante que elas não passem tempo excessivo em frente à televisão, mas dediquem tempo a brincadeiras e outras atividades lúdicas e educativas, pois assim, além de aprenderem, estarão menos suscetíveis às influências negativas exercidas pela mídia e terão mais possibilidade de manter o peso corporal e a saúde.

A combinação desses e outros aspectos conduz à formação do perfil nutricional da criança, sendo este o objetivo do trabalho, a partir da análise de crianças menores de cinco anos que frequentaram um berçário institucional em Brasília.

\section{Metodologia}

Foi realizada revisão de literatura com coleta de artigos nas bases de dados SciELO, Medline e LILACS, considerando os anos de 2000 a 2014. Foram utilizados os termos: aleitamento materno, consumo alimentar, influência da mídia e perfil nutricional. Foram selecionados artigos nas línguas inglesa e portuguesa. Inicialmente, os artigos foram filtrados pela relevância do título; após, foram lidos os resumos, e aqueles que tiveram informações pertinentes à revisão bibliográfica foram selecionados para leitura completa. Após a leitura completa, foram selecionados os artigos com relevância para integrarem a revisão. Agruparam-se então as informações semelhantes encontradas em cada um, a fim de se produzir um resumo crítico sobre cada tópico em questão.

Trata-se de um estudo transversal, em que o público-alvo foi composto das mães com crianças menores de cinco anos que frequentaram um berçário institucional do Distrito Federal. O ingresso das crianças no berçário ocorreu por volta de quatro a seis meses de idade, e o desligamento 
com um ano a um ano e três meses. A escolha do berçário foi por conveniência e a amostra foi universal. A amostra foi composta por crianças que frequentaram o berçário institucional escolhido no período de $1^{\circ}$ de março de 2010 (quando o berçário foi inaugurado) até 20 de dezembro de 2013, período anterior ao início da coleta de dados, totalizando 77 mães e 82 crianças. No total, 66 mães receberam o e-mail, pois 11 e-mails retornaram para a caixa de mensagem.

Foram incluídas as crianças que frequentaram o berçário e cujas mães continuavam a trabalhar no órgão público. Como critério de exclusão, determinou-se que as mães que não trabalham mais na instituição não teriam seus filhos participantes na pesquisa.

O primeiro contato foi feito por e-mail, disponibilizado pela equipe do berçário, a fim de explicar a pesquisa e convidar as mães para participar do estudo de forma presencial ou via e-mail, de acordo com a preferência de cada uma.

O encontro presencial ocorreu dia 21 de fevereiro de 2014 na própria instituição. Foram enviados e-mails com antecedência (dia 06 de fevereiro) e foi mantido contato com as mães que preferiram o encontro presencial, com o intuito de lembrá-las da data da coleta. Dezessete mães compareceram ao encontro presencial.

A coleta via e-mail foi realizada entre os dias 24 de fevereiro e 21 de março de 2014. Todas as orientações foram detalhadas e enviadas com os questionários, e eventuais dúvidas foram respondidas via e-mail. Nove mães participaram respondendo aos questionários por e-mail.

Tanto presencialmente quanto via e-mail, foi entregue um Termo de Consentimento Livre e Esclarecido que explicava a pesquisa e garantia o sigilo da identidade dos participantes. Após o aceite, foram entregues os instrumentos de coleta (dois questionários autoaplicáveis).

O primeiro questionário foi desenvolvido com o auxílio da equipe de nutricionistas do berçário local. Possuía 28 questões e as variáveis abordadas foram socioeconômicas (estado civil, escolaridade, renda familiar, número de pessoas residentes no domicílio), ambientais (se a criança costuma fazer as refeições assistindo à televisão), demográficas (idade materna, idade da criança, número de filhos), biológicas (tipo de parto, peso ao nascer, peso pré-gestacional, idade gestacional, recebimento de orientações sobre alimentação complementar) e antropométricas (peso e altura ao sair do berçário e atuais). Todas as questões eram objetivas ou possuíam um campo em branco para a mãe escrever o que era pedido, como peso e altura da criança.

Para avaliação da adequação do questionário, antes de sua aplicação na instituição, foi realizado um teste piloto com sete mães que não participariam da pesquisa, mas possuíam características semelhantes ao grupo estudado (idade, escolaridade, renda familiar e idade dos filhos). Não houve necessidade de alteração do material. 
Para a avaliação dos resultados, as crianças foram divididas em dois grupos etários (1-3 anos e 4-5 anos), segundo a divisão apresentada pela Sociedade Brasileira de Pediatria, ${ }^{12}$ conforme mostra a tabela 1. A partir de então, foram analisados aspectos do consumo alimentar, estado nutricional e o score de alimentação saudável.

Tabela 1. Recomendação de porções diárias de alimentos por faixa etária segundo a Sociedade Brasileira de Pediatria ${ }^{12}$. Brasil, 2013.

\begin{tabular}{lcccc}
\hline Grupo alimentar & $6-11$ meses & $1-3$ anos & $\begin{array}{c}\text { Pré-escolar }(4 \\
-8 \text { anos })\end{array}$ & $\begin{array}{c}\text { Adolescentes e } \\
\text { adultos }\end{array}$ \\
\hline Arroz & 3 & 5 & 5 & 5 a 9 \\
Feijão & 1 & 1 & 1 & 1 \\
Verdura & 3 & 3 & 3 & 4 a 5 \\
Fruta & 4 & 3 & 4 & 4 a 5 \\
Carne & 2 & 2 & 2 & 1 a 2 \\
Leite e derivados & Leite materno & 3 & 3 & 3 \\
Açúcar e doces & 0 & 1 & 1 & 1 a 2 \\
Óleos e gorduras & 2 & 2 & 1 & 1 a 2 \\
\hline
\end{tabular}

A avaliação do consumo alimentar habitual das crianças foi realizada por meio de um questionário de frequência alimentar (QFA) qualitativo validado para crianças de dois a cinco anos de idade. O QFA possui 57 itens alimentares, divididos em dez categorias, sendo elas: cereais (arroz, pão, batata frita, macarrão instantâneo); leguminosas; hortaliças; frutas (frutas em geral e suco de frutas); carnes e ovos; leite e derivados; açúcar e doces; salgados e preparações (pizza, misto quente, hambúrguer); bebidas açucaradas; e outros. ${ }^{13}$

Para a avaliação desses dados, foi utilizado um escore de alimentação saudável baseado no Recommended Foods Score (Contagem de Alimentos Recomendados - CAR), segundo o qual cada alimento da lista de alimentos recomendados utilizada equivaleria a um ponto. Esse método de pontuação foi desenvolvido por Kant et al., ${ }^{14}$ e McCullough et al., ${ }^{15}$ com o intuito de verificar a qualidade global da dieta por meio de um QFA. A lista de alimentos recomendados foi composta por 28 alimentos, sendo portanto a pontuação máxima adquirida por cada criança equivalente a 28 pontos. 
No presente estudo, a Contagem de Alimentos Recomendados foi realizada segundo a metodologia desses autores e foi adaptada às recomendações da Sociedade Brasileira de Pediatria, ${ }^{12}$ conforme mostra a tabela 1, que é dividida em arroz, feijão, verdura, fruta, carne, leite e derivados, açúcar e doces, e óleos e gorduras. Para que a classificação fosse o mais fiel possível, o grupo de bebidas açucaradas do QFA foi incluído no grupo de açúcares e doces.

Como forma de agradecimento pela participação das mães, foi entregue um material desenvolvido com o auxílio da nutricionista do local, contendo: orientações para uma alimentação saudável, proposta de cardápio de um mês e receitas saudáveis para oferecer de lanche para as crianças.

Os dados foram analisados por meio dos programas Excel®, AntrhoPlus (versão 1.0.4) e Statistical Package for the Social Sciences - SPSS ${ }^{\circledR}$ (versão 21) para análises estatísticas descritivas: medidas de tendência central (média) e medidas de dispersão (desvio padrão), além de classificação do estado nutricional.

O presente projeto foi aprovado pelo Comitê de Ética em Pesquisa da Faculdade de Ciências da Saúde da Universidade de Brasília, parecer nº 394.040.

\section{Resultados}

O convite para participar da pesquisa foi enviado para 66 mães, das quais 26 responderam (39\%), sendo nove respostas via e-mail e 17 presenciais. Dentre as participantes, duas possuíam dois filhos, totalizando 28 crianças.

A maioria das mães (67,9\%) se encontra na faixa etária de 31 a 40 anos; 85,7\% estão eutróficas e 14,3\% com sobrepeso. Em relação ao tipo de parto e número de filhos, $75 \%$ tiveram parto cesáreo, $53,6 \%$ possuem apenas um filho e $28,6 \%$ possuem dois filhos. A maioria é casada (75\%), possui pós-graduação (67,9\%) e recebe mais de dez salários mínimos mensais (85,7\%). A amostra das crianças teve a média de idade de 29,44 meses $\pm 14,18$, sendo $60,7 \%$ do sexo feminino. O tempo médio de permanência no berçário foi de 6,43 meses.

Permaneceram em aleitamento materno exclusivo (AME) até os seis meses $67,9 \%$ das crianças; $50 \%$ foram desmamadas entre 12 e 15 meses e 7,1\% foram desmamadas entre 21 e 24 meses, conforme mostra a tabela 2 . 
Tabela 2. Prevalência de aleitamento materno, desmame e tipo de orientação para introdução alimentar em crianças que frequentaram um berçário institucional entre 2010 e 2013, Brasília-DF.

\begin{tabular}{|c|c|c|}
\hline Variável & $\mathrm{n}$ & $\%$ \\
\hline \multicolumn{3}{|l|}{ Permanência em AME } \\
\hline AME até 1 mês & 1 & 3,6 \\
\hline AME até 4 meses & 2 & 7,1 \\
\hline AME até 5 meses & 5 & 17,9 \\
\hline AME até 6 meses & 19 & 67,9 \\
\hline AME por mais de 6 meses & 1 & 3,6 \\
\hline \multicolumn{3}{|l|}{ Período de Desmame } \\
\hline Desmame entre 3 e 6 meses incompletos & 3 & 10,7 \\
\hline Desmame entre 6 e 9 meses incompletos & 1 & 3,6 \\
\hline Desmame entre 9 e 12 meses incompletos & 5 & 17,9 \\
\hline Desmame entre 12 e 15 meses incompletos & 14 & 50,0 \\
\hline Desmame entre 15 e 18 meses incompletos & 0 & - \\
\hline Desmame entre 18 e 21 meses incompletos & 0 & - \\
\hline Desmame entre 21 e 24 meses incompletos & 2 & 7,1 \\
\hline Desmame entre 24 e 27 meses incompletos & 2 & 7,1 \\
\hline Desmame entre 27 e 30 meses incompletos & 1 & 3,6 \\
\hline \multicolumn{3}{|l|}{ Orientou para introdução alimentar } \\
\hline Pediatra & 9 & 32,1 \\
\hline Nutricionista & 9 & 32,1 \\
\hline Pediatra e nutricionista & 9 & 32,1 \\
\hline Familiares / amigos & 1 & 3,6 \\
\hline
\end{tabular}


Em relação ao estado nutricional, todas as crianças, dos dois grupos etários, foram classificadas com peso e estatura adequados para a idade. Do grupo de crianças de um a três anos, 87\% (20 indivíduos) foram classificados com eutrofia e 13\% (3 indivíduos) com risco de sobrepeso; e do grupo de crianças de 4-5 anos, todas foram classificadas com eutrofia segundo a curva de peso por estatura. ${ }^{16}$

Os resultados obtidos do Questionário de Frequência Alimentar (QFA) indicaram que a maioria das crianças, dos dois grupos etários, estava com o consumo de cereais (1-3 anos, 70\% e 4-5 anos, $100 \%$ ), hortaliças (1-3 anos, 66\% e 4-5 anos, 75\%), e salgados (1-3 anos, 100\% e 4-5 anos, 100\%) abaixo do recomendado. O grupo das carnes, para o grupo de 1-3 anos (62\%); o grupo dos leites, para o grupo de 4-5 anos (75\%); e bebidas açucaradas, para ambos os grupos (100\%), apresentou consumo adequado. Já o grupo das leguminosas, para o grupo de 1-3 anos (54\%) mostrou-se com consumo acima, de acordo com a tabela 3. Além disso, foi verificado que, do total de crianças, 10,7\% tinham hábito de realizar as refeições assistindo à televisão quase sempre ;e 53,5\%, nunca ou raramente.

Tabela 3. Consumo atual dos grupos alimentares segundo o Questionário de Frequência Alimentar qualitativo de crianças que frequentaram um berçário institucional entre 2010 e 2013, Brasília-DF, 2013.

\begin{tabular}{ccccc}
\hline Variável & $\mathrm{n}$ & $\mathrm{n}$ & $\mathrm{n}$ & $\%$ \\
& $1-3$ anos & $4-5$ anos & Total & Total \\
\hline Consumo de Cereais & & & & \\
Abaixo do recomendado & 17 & 4 & 21 & 75 \\
Adequado & 2 & 0 & 2 & 7,1 \\
Acima do recomendado & 5 & 0 & 5 & 17,9 \\
\hline Consumo de Leguminosas & & & 6 & 21,4 \\
Abaixo do recomendado & 4 & 2 & 8 & 28,6 \\
Adequado & 7 & 1 & 14 & 50,0 \\
Acima do recomendado & 13 & 1 & & \\
\hline Consumo de Frutas & & & 9 & 32,2 \\
Abaixo do recomendado & 8 & 1 & 8 & 28,6 \\
Adequado & 7 & 2 & 11 & 39,3 \\
Acima do recomendado & 9 & 1 & & \\
\hline
\end{tabular}




\begin{tabular}{|c|c|c|c|c|}
\hline Variável & $\begin{array}{c}\mathrm{n} \\
1-3 \text { anos }\end{array}$ & $\begin{array}{c}\mathrm{n} \\
4-5 \text { anos }\end{array}$ & $\begin{array}{c}\mathrm{n} \\
\text { Total }\end{array}$ & $\begin{array}{c}\% \\
\text { Total }\end{array}$ \\
\hline \multicolumn{5}{|l|}{ Consumo de Hortaliças } \\
\hline Abaixo do recomendado & 16 & 3 & 19 & 67,9 \\
\hline Adequado & 5 & 1 & 6 & 21,4 \\
\hline Acima do recomendado & 3 & 0 & 3 & 10,7 \\
\hline \multicolumn{5}{|l|}{ Consumo de Carnes e Ovos } \\
\hline Abaixo do recomendado & 5 & 2 & 7 & 28,6 \\
\hline Adequado & 15 & 2 & 17 & 60,7 \\
\hline Acima do recomendado & 4 & 0 & 4 & 7,1 \\
\hline \multicolumn{5}{|l|}{ Consumo de Leite e Derivados } \\
\hline Abaixo do recomendado & 15 & 1 & 16 & 57,1 \\
\hline Adequado & 7 & 3 & 10 & 35,7 \\
\hline Acima do recomendado & 2 & 0 & 2 & 7,1 \\
\hline \multicolumn{5}{|l|}{$\begin{array}{l}\text { Consumo de açúcar, doces e } \\
\text { bebidas açucaradas }\end{array}$} \\
\hline Abaixo do recomendado & 10 & 2 & 12 & 42,9 \\
\hline Adequado & 7 & 1 & 8 & 28,6 \\
\hline Acima do recomendado & 7 & 1 & 8 & 28,6 \\
\hline
\end{tabular}

Quanto à pontuação para avaliar a qualidade global da dieta, foi observada uma média 23,58 pontos para o grupo de crianças de 1-3 anos e de 21,25 pontos para o grupo de crianças de 4-5 anos, de um total de 28 pontos, o que indica, de acordo com a metodologia utilizada, que as crianças, de forma geral, possuem uma alimentação saudável.

\section{Discussão}

O perfil das mães foi bem homogêneo, sendo semelhantes características como idade, escolaridade, renda familiar e estado civil. O alto nível de escolaridade e renda familiar das mães representa um fator positivo em relação à alimentação e ao estado nutricional dos filhos, pois 
quanto mais acesso à informação, mais fácil é o processo de educação e conscientização alimentar e nutricional, conforme observado no estudo de Castro et al., ${ }^{17} \mathrm{em}$ que a escolaridade e a renda familiar se mostraram como fatores que podem contribuir para a melhora do estado de saúde, de uma forma geral.

Em relação às características antropométricas das mães, constatou-se que a maioria foi classificada como eutrófica, enquanto 14,3\% estavam com sobrepeso. Segundo a Pesquisa de Orçamento Familiar (POF), ${ }^{18} 48 \%$ das mulheres estava com sobrepeso, diferentemente dos dados apresentados nesta pesquisa, em que a maioria se encontra com o estado nutricional adequado. Os dados socioeconômicos identificados na referida pesquisa de Castro et al. ${ }^{17}$ podem explicar, ao menos parcialmente, a diferença entre os resultados do presente estudo e da POF. ${ }^{18}$

Quanto ao tipo de parto e número de filhos, foi constatado que a maioria das mães teve parto cesáreo (75\%), número ainda menor do que o encontrado em estudo realizado em São Luís do Maranhão, segundo o qual 97,8\% dos partos realizados em maternidades privadas foram cesáreos. ${ }^{19}$ Já no Relatório Nacional de Acompanhamento de maio de $2014,{ }^{20}$ verificou-se que quase $54 \%$ dos partos eram cesáreos, sendo que a Organização Mundial da Saúde $(\mathrm{OMS})^{21}$ recomenda que a porcentagem fique entre 5 a $15 \%$, com o intuito de diminuir os riscos causados pela realização indiscriminada de cesáreas. Sendo assim, o presente estudo apresenta percentual de cesáreas mais elevado do que o recomendado pela OMS. ${ }^{21}$

Em relação ao número de filhos, foi verificada maior prevalência de um filho por mulher. De acordo com o Censo Demográfico do Instituto Brasileiro de Geografia e Estatística (IBGE), ${ }^{22}$ a fecundidade total no Brasil em 2010 chegou a 1,9 filho, ficando abaixo do nível de reposição da população, que seria de 2,1 filhos. Isso revela que a média do número de filhos apresentada na pesquisa está abaixo do recomendado para a reposição da população. Entretanto, a maioria das mulheres ainda se encontra em período fértil, podendo vir a ter mais filhos tardiamente.

Os cruzamentos de dados realizados entre as mães e as crianças não geraram resultados com significância, o que pode se dever ao tamanho da amostra analisada. Mais estudos referentes a esses temas, com uma amostra maior, podem ser importantes para a análise de possíveis associações.

Quanto às crianças, observou-se elevado número que permaneceu em aleitamento materno exclusivo até os seis meses de idade, não sendo encontrada diferença entre os grupos etários, de modo semelhante ao encontrado na pesquisa sobre o Aleitamento Materno nas Capitais Brasileiras, ${ }^{5}$ em que $50 \%$ das crianças do Distrito Federal permaneceram em aleitamento exclusivo até os seis meses. Porcentagem diferente foi verificada no estudo de Brecailo et al., ${ }^{4}$ realizado com 426 crianças entre 0 e 23,9 meses em famílias de baixa renda, em que 22,4\% da amostra permaneceram em aleitamento materno exclusivo até os quatro meses e apenas 12,9\% até os seis. No mesmo estudo, foi verificado que as mães que trabalhavam fora de casa interrompiam mais precocemente o aleitamento exclusivo antes de a criança completar o sexto mês de vida. Destaca-se, na discussão do 
referido artigo, que a inexistência de creche no local de trabalho da mãe e a licença-maternidade inferior a seis meses foram fatores determinantes para o desmame precoce.

O estudo de Simon et al. ${ }^{23}$ também mostrou que há maior risco de sobrepeso e obesidade em crianças que possuem mães que trabalham fora de casa, pelo fato de não acompanharem o que é ingerido durante o dia e algumas vezes quererem compensar sua ausência presenteando a criança com alimentos altamente energéticos. Portanto, a licença-maternidade de seis meses pode favorecer o aleitamento exclusivo, em razão da proximidade da criança em relação à mãe durante todo o período recomendado.

Após os seis meses de idade, deve ser iniciada a alimentação complementar associada ao aleitamento materno, padrão que deve perdurar até os dois anos de idade da criança ou mais. No presente estudo, foi verificado que 67,8\% das crianças receberam leite materno até os 12 meses. Essa elevada prevalência pode ser justificada pela presença do berçário no local de trabalho da mãe. Na pesquisa sobre o Aleitamento Materno nas Capitais Brasileiras, ${ }^{5}$ averiguou-se resultado semelhante, com prevalência do aleitamento materno até um ano de idade no Distrito Federal de $65,4 \%$, mas a maioria das mães não trabalhava fora de casa.

É importante ressaltar que entre 12 e 15 meses, 50\% da amostra foram desmamados, correspondendo ao mesmo período em que ocorre o desligamento do berçário. Portanto, é possível que haja associação entre o desligamento do berçário e o período do desmame. Pode ser interessante que o berçário passe a atender as crianças até dois anos de idade, com o intuito de incentivar a manutenção da amamentação. Esse incentivo é essencial, pois como a criança está passando por uma fase de adaptação com novos alimentos, texturas e sabores, nem sempre terá plena aceitação nos primeiros contatos, e o leite materno auxilia na prevenção de possíveis carências e deficiências nutricionais. ${ }^{24}$

Já em relação ao recebimento de orientação para a introdução alimentar, constatou-se que $35,7 \%$ da amostra obtiveram orientações provenientes dos pediatras, familiares e amigos, e 64,2\%, de nutricionistas. Trata-se de uma realidade positiva, uma vez que, sendo atribuição privativa do nutricionista a prescrição de dietas, ${ }^{25}$ verifica-se sua importância nessa fase de introdução alimentar, para que haja a prescrição correta da melhor forma de iniciar os alimentos para a criança.

O estado nutricional das crianças em relação à curva de peso para idade e estatura para idade foi classificado como eutrófico. De acordo com a curva de peso por estatura, 88,9\% foram classificadas como eutróficas e 11,1\%, com risco de sobrepeso, sendo que no grupo de 4-5 anos todos estavam com eutrofia, mas no grupo de 1-3 anos, 86,9\% estavam com eutrofia e 13,1\% com risco de sobrepeso. Segundo Sperandio et al., ${ }^{26}$ em estudo transversal realizado em oito cidades brasileiras com participação de 350 crianças, de ambos os sexos, com 2-5 anos de idade, $66 \%$ estavam com peso adequado para a idade, $83,5 \%$ com estatura adequada para a idade, 10,8\% com baixopeso, $63,5 \%$ com eutrofia e $25,8 \%$ com risco de sobrepeso, conforme a curva de peso por estatura. 
A transição nutricional apresentou esse novo perfil de diminuição da desnutrição e aumento do sobrepeso e da obesidade, como verificado nos dados apresentados acima. O excesso de peso predispõe as crianças a diversas doenças crônicas e respiratórias, que podem causar problemas em seu desenvolvimento e crescimento. Portanto, é imprescindível o diagnóstico precoce, pois quanto mais cedo for a descoberta, mais fácil será o tratamento. ${ }^{27}$

Em paralelo, verificou-se que 46,4\% das crianças têm costume de realizar as refeições assistindo à televisão às vezes ou quase sempre e $53,5 \%$, nunca ou raramente. De fato, a mídia exerce influência sobre as escolhas alimentares das crianças, levando-as a preferir alimentos com maior densidade energética e menor concentração de vitaminas e minerais. No estudo de Fiates et al., ${ }^{28}$ realizado com 57 escolares de 7-10 anos em uma escola particular, foi observado que a maioria da amostra costumava assistir à televisão durante as refeições (93,1\% das mulheres e 85,7\% dos homens). Na pesquisa bibliográfica realizada, não foram encontrados artigos que comparassem crianças com a mesma faixa etária do presente estudo. A partir desses dados, verifica-se a possível necessidade de uma intervenção para que os pais fiquem cientes do elevado número de crianças que consomem suas refeições assistindo à televisão e do prejuízo que isso pode acarretar para a formação de seus hábitos alimentares e seu estado nutricional, como aumento do sedentarismo e predisposição para o desenvolvimento de sobrepeso e obesidade.

O consumo alimentar foi analisado no presente estudo por meio do Questionário de Frequência Alimentar qualitativo validado para crianças de 2-5 anos de idade. Depender da memória e da colaboração do indivíduo, além do fato de alguns alimentos não serem bem especificados (como no caso das sopas infantis, que não eram classificadas como industrializadas ou naturais), pode gerar dúvidas na hora do preenchimento. Entretanto, este inquérito foi escolhido por ser de fácil, simples e rápida aplicação, baixo custo, não influenciar ou alterar o padrão alimentar e fornecer a análise do consumo habitual das crianças. ${ }^{29,30}$

De acordo com o Questionário de Frequência Alimentar, foi verificado o consumo de alguns grupos alimentares acima do recomendado pela Sociedade Brasileira de Pediatria, ${ }^{12}$ e a ingestão de outros, abaixo.

O consumo elevado de frutas pode decorrer do fato de que o QFA utilizado classificou o suco de fruta natural dentro do grupo das frutas. Entretanto, esta classificação não é a mais indicada, pois estudos realizados com crianças mostraram que o consumo excessivo de suco da fruta pode levar a um aumento da obesidade. Sanigorski et al. ${ }^{31}$ realizaram estudo com crianças de 4-12 anos de idade e verificaram que aquelas que ingeriam suco frequentemente ( 2 vezes por dia ou mais) estavam mais suscetíveis a ficar com sobrepeso e obesidade se comparadas a crianças que consumiam menos. Conclusões semelhantes foram encontrados na pesquisa de Myles et al., ${ }^{32}$ segundo a qual, em relação às crianças que estavam com risco de sobrepeso, o aumento do consumo de suco de frutas estava associado ao aumento da adiposidade, enquanto o consumo de frutas estava ligado 
a sua diminuição. Assim, verifica-se a importância de classificar as frutas e os sucos em grupos separados, e não em um grupo único, como fez o Questionário de Frequência Alimentar utilizado.

Quanto ao consumo de leguminosas, há pesquisas que indicam um consumo adequado ou abaixo da recomendação, como a de Barbosa et al., ${ }^{33}$ contrapondo-se aos resultados do presente estudo, em que o consumo da maioria estava acima do recomendado (provavelmente, pelo fato de ser orientada, pelos nutricionistas do local, a ingestão no almoço e no jantar). Sendo assim, percebe-se a necessidade de realizar mais estudos para verificar se a recomendação de uma porção por dia é suficiente para suprir as necessidades da criança nesse período da vida, visto que o consumo inadequado pode contribuir para o desenvolvimento de carências nutricionais, como a deficiência de ferro. ${ }^{34}$

Já o consumo de carnes mostrou-se adequado para a maioria das crianças, diferentemente do encontrado no estudo realizado por Bortolini et al., ${ }^{9}$ que mostrou que $38,2 \%$ das crianças de 6-59 meses consumiam carne, frango ou peixe diariamente, estando com a ingestão abaixo da verificada no presente estudo. Esse grupo alimentar é importante e sua ingestão precisa ser estimulada, pois possui boa disponibilidade de ferro heme e outros nutrientes importantes para o crescimento e desenvolvimento.

O consumo dos cereais mostrou-se abaixo do recomendado, o que talvez decorra do fato de que o QFA utilizado incluía nesse grupo biscoitos recheados, batata frita e macarrão instantâneo, por exemplo, alimentos que muitas mães relataram não oferecer a seus filhos. Na pesquisa de Barbosa et al., ${ }^{33}$ também se verificou consumo abaixo do recomendado, porém grande parte do consumo desse grupo era proveniente dos biscoitos salgados e recheados.

A ingestão de leite também se mostrou abaixo do recomendado. Contudo, no questionário entregue às mães, não houve nenhuma pergunta abordando a existência de alguma intolerância ou alergia, e este pode ser um dos fatores para o resultado encontrado quanto à ingestão desse grupo alimentar. Ademais, o questionário não abordou o consumo do leite materno como fonte de leite. Na pesquisa de S. Filha et al., ${ }^{34}$ também foi verificado o consumo abaixo do recomendado. Deve-se dar atenção ao consumo destes alimentos, pois são ricas fontes de cálcio, mineral importante para a saúde óssea, pois contribui com a formação, mineralização e rigidez dos ossos. ${ }^{35}$

O baixo consumo de hortaliças foi verificado em $67,9 \%$ das crianças, enquanto $32,1 \%$ delas apresentaram consumo adequado ou acima do recomendado. No estudo de Alves et al., ${ }^{36} \mathrm{e}$ Bortolini et al., ${ }^{9}$ também se verificou baixo consumo de hortaliças. Por serem alimentos ricos em fibras, vitaminas e minerais, a ingestão desse grupo deve ser estimulada. No caso específico do público infantil, é interessante usar a criatividade para inovar, oferecer receitas novas e investir na apresentação do prato, pois condutas como essas chamam a atenção da criança e podem despertála para que aumente o consumo em relação a esses alimentos. 
O consumo de açúcares e bebidas açucaradas representou-se 42,9\% abaixo do recomendado. Um estudo realizado em Aracaju com crianças de 6-35 meses mostrou resultado diferente. Naquele caso, a maioria das crianças tinha excessivo consumo de açúcares e doces. ${ }^{34}$ Muitos artigos mostram a relação do consumo excessivo de doces e açúcares com sobrepeso, obesidade e outras doenças crônicas - portanto, a ingestão desse grupo alimentar deve ser evitada e a oferta para as crianças deve ocorrer mais tarde. ${ }^{23}$

Quanto ao grupo de salgados e preparações (pizza, hambúrguer, etc.), foi verificado que 42,9\% das crianças consumiam tais alimentos pelo menos uma vez ao dia. Entretanto, a ingestão desses alimentos não deve ser estimulada, pois eles estão relacionados ao risco de aumento do sobrepeso e obesidade. Portanto, o ideal seria um consumo eventual, e não diário.

Assim, apesar da variação na ingestão de alguns grupos alimentares, foi verificado que a nota global média para avaliação de uma dieta saudável foi alta: 23,50 pontos para o grupo de 1-3 anos e 21,25 pontos para o grupo de 4-5 anos, de um total de 28 pontos. No estudo de Coelho et al., ${ }^{37}$ que utilizou a mesma metodologia, mas um Questionário de Frequência Alimentar distinto, a média do escore para crianças de 6-9 anos foi de $16,00 \pm 6,83$ pontos, de um total de 50 pontos. Ou seja, é possível perceber que a média das crianças no presente estudo se mostrou elevada, o que pode sugerir uma influência positiva do berçário em sua alimentação.

\section{Conclusão}

Conclui-se que mais de dois terços da amostra permaneceram em aleitamento materno exclusivo até os seis meses, e metade das crianças foi desmamada entre a idade de um ano e um ano e três meses, mesmo período em que há o desligamento do berçário.

Quanto ao consumo alimentar das crianças, constatou-se déficit na ingestão de hortaliças, alimentos ricos em nutrientes e fibras que são importantes para o desenvolvimento saudável das crianças. O consumo mediano de alimentos com alta densidade energética foi encontrado em quase $43 \%$ da amostra, o que não deveria ser estimulado, porque eles proporcionam a ingestão excessiva de calorias em detrimento dos minerais, o que é prejudicial à saúde e ao crescimento adequado. O consumo de leguminosas mostrou-se elevado para $50 \%$ da amostra, e o de frutas, para $40 \%$. Esses resultados se revelam positivos, por se tratar de alimentos ricos em nutrientes e que contribuem para o bom desenvolvimento da criança.

Ressalta-se a importância da realização de novos estudos utilizando inquéritos de consumo alimentar, de base populacional, que impulsionem o monitoramento nutricional das crianças e utilizem metodologia ajustada a esse modelo de interpretação. 
De toda forma, espera-se que esses resultados deem subsídios para que se avaliem os efeitos a longo prazo dos trabalhos desenvolvidos nos berçários, como o incentivo ao aleitamento materno, a criação de bons hábitos alimentares e a prevenção do sobrepeso, com o intuito de atender melhor às necessidades da população menor de cinco anos.

\section{Referências}

1. Instituto Brasileiro de Geografia e Estatística. Censo demográfico 2010: educação e deslocamento. Rio de Janeiro: IBGE; 2010. [acesso em 18 nov. 2013]. Disponível em: ftp://ftp.ibge.gov.br/Censos/ Censo_Demografico_2010/Educacao_e_Deslocamento/censo_educacao_e_deslocamento.pdf

2. Bartoshuk LM. Comparing sensory experiences across individuals: Recent psychophysical advances illuminate genetic variation in taste perception. Chem. Senses. 2000; 25(4):447-460.

3. Schwartz C, Scholtens PAMJ, Lalanne A, Weenen H, Nicklaus S. Development of healthy eating habits early in life. Review of recent evidence and selected guidelines. Appetite 2011; 57(3):796-807.

4. Brecailo MK, Corso ACT, Almeida CCB, Schmitz BAS. Fatores associados ao aleitamento materno exclusivo em Guarapuava, Paraná. Rev. Nutr. [Internet]. 2010 2014; 23(4):553-563.

5. Brasil. Ministério da Saúde. II Pesquisa de Prevalência de Aleitamento Materno nas Capitais Brasileiras e Distrito Federal. Brasília: Ministério da Saúde; 2009.

6. Venancio SI, Escuder MML, Saldiva SRDM, Giugliani ERJ. A prática do aleitamento materno nas capitais brasileiras e Distrito Federal: situação atual e avanços. J. Pediatr. 2010; 86(4):317-324.

7. Viana V, Santos PL, Guimarães MJ. Comportamento e hábitos alimentares em crianças e jovens: uma revisão da literatura. Psic. Saúde Doenças 2008; 9(2):209-231.

8. Broilo MC, Louzada MLC, Drachler ML, Stenzel LM, Vitolo MR. Percepção e atitudes maternas em relação às orientações de profissionais de saúde referentes a práticas alimentares no primeiro ano de vida. J. Pediatr. 2013; 89(5):485-491.

9. Bortolini GA, Gubert MB, Santos LMP. Consumo alimentar entre crianças brasileiras com idade de 6 a 59 meses. Cad. Saúde Pública 2012; 28(9):1759-1771.

10. Batista FM, Souza AI, Miglioli TC, Santos MC. Anemia e obesidade: um paradoxo da transição nutricional brasileira. Cad. Saúde Pública 2008; 24(Suppl 2):S247-S257.

11. Motta-Gallo S, Gallo P, Cuenca A. Influência da televisão nos hábitos alimentares de crianças do nordeste brasileiro. Rev. Bras. Crescimento Desenvolv. Hum. 2013; 23:89-93.

12. Sociedade Brasileira de Pediatria. Manual de orientação para a alimentação do lactente, do pré-escolar, do escolar, do adolescente e na escolar. $3^{\text {a }}$. ed. Rio de Janeiro: SBP, Departamento de Nutrologia; 2012.

13. Colucci ACA, Philippi ST, Slater B. Desenvolvimento de um questionário de freqüência alimentar para avaliação do consumo alimentar de crianças de 2 a 5 anos de idade. Rev. Bras. Epidemiol. 2004; 7(4): 393-401. 
14. Kant AK, Schatzkin A, Graubard BI, Schairer C. A prospective study of diet quality and mortality in women. JAMA 2000; 283(16):2109-15.

15. McCullough ML, Feskanich D, Stampfer MJ, Giovannucci EL, Rimm EB, Hu FB, et al. Diet quality and major chronic disease risk in men and women: moving toward improved dietary guidance. Am. J. Clin. Nutr. 2002; 76(6):1261-71.

16. World Health Organization. Who child growth standards: length/height-for-age, weight-for-age, weight-for-lenght, weight-for-height and body mass index-for-age. Methods and development. Geneva: WHO; 2006.

17. Castro TG, Novaes JF, Silva MR, Costa NMB, Franceschini SCC, Tinôco ALA, et al. Caracterização do consumo alimentar, ambiente socioeconômico e estado nutricional de pré-escolares de creches municipais. Rev. Nutr. 2005; 18(3):321-330.

18. Instituto Brasileiro de Geografia e Estatística. Pesquisa de orçamentos familiares 2008-2009: antropometria e estado nutricional de crianças, adolescentes e adultos no Brasil. Rio de Janeiro: IBGE; 2010. 122 p.

19. Mandarino NR, Chein MBC, Monteiro Junior FC, Brito LMO, Lamy ZC, Nina VJS, et al. Aspectos relacionados à escolha do tipo de parto: um estudo comparativo entre uma maternidade pública e outra privada, em São Luís, Maranhão, Brasil. Cad. Saúde Pública 2009; 25(7):1587-1596.

20. Instituto de Pesquisa Econômica Aplicada. Objetivos de desenvolvimento do milênio. Relatório nacional de acompanhamento. Brasília: Ipea, Secretaria de Planejamento e Investimento Estratégico; 2014. 208 p.

21. Organização Mundial de Saúde. Assistência ao parto normal: um guia prático: relatório de um grupo técnico. Genebra: OMS; 1996. 53 p.

22. Instituto Brasileiro de Geografia e Estatística. Censo demográfico 2010: nupcialidade, fecundidade e migração. Resultados da amostra. Rio de Janeiro: IBGE; 2010. 349 p.

23. Simon VGN, Souza JMP, Souza SB. Aleitamento materno, alimentação complementar, sobrepeso e obesidade em pré-escolares. Rev. Saúde Pública 2009; 43(1):60-69.

24. Dias MCA, Freire LMS, Franceschini SCC. Recomendações para alimentação complementar de crianças menores de dois anos. Rev. Nutr. 2010; 23(3):475-486.

25. Brasil. Lei no 8.234, de 17 de setembro de 1991. Regulamenta a profissão de nutricionista e determina outras providências. Diário Oficial da União. 18 set. 1991.

26. Sperandio N, Sant'Ana LFR, Franceschini SCC, Priore SE. Comparação do estado nutricional infantil com utilização de diferentes curvas de crescimento. Rev. Nutr. 2011; 24(4):565-574.

27. Adami F, Vasconcelos FAG. Childhood and adolescent obesity and adult mortality: a systematic review of cohort studies. Cad. Saúde Pública 2008; 24(Suppl 4): S558-S568.

28. Fiates GMR, Amboni RDMC, Teixeira E. Comportamento consumidor, hábitos alimentares e consumo de televisão por escolares de Florianópolis. Rev. Nutr. 2008; 21(1):105-114.

29. Molina MCB, Lopéz PM, Faria CP, Cade NV, Zandonade E. Predictivos socioeconómicos de lacalidad de laalimentación de niños. Rev. Saúde Pública 2010; 44(5):785-732. 
30. Anjos LA, Souza DR, Rossato SL. Desafios na medição quantitativa da ingestão alimentar em estudos populacionais. Rev. Nutr. 2009; 22(1):151-161.

31. Sanigorski AM, Bell AC, Awinburn BA. Association of key foods and beverages with obesity in Australian schoolchildren. Public Health Nutr. 2007;10:152-157.

32. Faith MS, Dennison BA, Edmunds LS, Stratton HH. Fruit juice intake predicts increased adiposity gain in children from low-income families: weight status-by-environment interaction. Pediatrics 2006; 118:2066-2075.

33. Barbosa RMS, Croccia C, Carvalho CGN, Franco VC, Salles-Costa R, Soares EA. Consumo alimentar de crianças com base 4 na pirâmide alimentar brasileira infantil. Rev. Nutr. 2005; 18(5):633-641.

34. S. Filha EO, Araújo JS, Barbosa JS, Gaujac DP, Santos CFS, Silva DG. Consumo dos grupos alimentares em crianças usuárias da rede pública de saúde do município de Aracaju, Sergipe. Rev. Paul. Pediatr. 2012; 30(4):529-536.

35. Bueno AL, Czepielewski MA. A importância do consumo dietético de cálcio e vitamina D no crescimento. J. Pediatr. 2008; 84(5):386-394.

36. Alves MN, Muniz LC, Vieira MFA. Consumo alimentar entre crianças brasileiras de dois a cinco anos de idade: Pesquisa Nacional de Demografia e Saúde (PNDS), 2006. Ciênc. Saúde Coletiva 2013; 18(11):3369-3377.

37. Coelho LG, Cândido APC, Machado-Coelho GLL, Freitas SN. Association between nutritional status, food habits and physical activity level in schoolchildren. J. Pediatr. 2012; 88(5):406-412.

Recebido: 17/12/2014

Revisado: 06/1/2015

Aprovado: $14 / 1 / 2015$ 